\title{
A PARAMETRIC METHOD FOR LINKING MEMS PACKAGE AND DEVICE MODELS
}

\author{
Andrew C. McNeil \\ Motorola Sensors Division, Mail Stop Z-207 \\ 5005 E. McDowell Rd., Phoenix, AZ, 85008 \\ e-mail: r16360@email.mot.com
}

\begin{abstract}
Package stresses can significantly affect the performance of MEMS devices. This paper presents a method of modeling the effect of package stresses on MEMS device output. This method uses two separate FEA models, one for the MEMS device and one for the package. The results of the package model are approximated by several (1-6) parameters, and are used as inputs to the MEMS device model. This technique can efficiently model package stresses. It also provides parameters to quantify package effects, allowing communication between MEMS package and device designers. Model results are compared to experimental data, and are shown to be in good agreement.
\end{abstract}

\section{INTRODUCTION}

Many potential MEMS applications require low cost packaging to meet aggressive cost targets [1], [2], [3]. As these packages often use materials with coefficients of thermal expansion higher than silicon (e.g. polymers, metals) significant thermal stresses often develop during manufacture or operation. These stresses can affect MEMS device output. Thus a method is needed to efficiently simulate the effect of package stresses on MEMS devices during both package and MEMS device development.

Where some previous work has used one large, packagedevice FEA model [4], it is often difficult to implement a single FEA model for both the package and the device. The device is usually much smaller than the package, and thus requires much higher mesh densities. Meshing the package with these high mesh densities would require an extremely large model (in some cases greater than a million nodes). Further, MEMS devices and packages are sometimes developed separately, and this makes it difficult to assemble a combined model.

The different scales of the package and device models can be accommodated by using two separate FEA models, a "fine mesh" model for the device and a "coarse mesh" model for the package. Because the package model is usually much more stiff $(\sim$ million $X)$ than the device, the package model can be run independently of the device model, and displacements from the package model can then be passed to the device model, where they are imposed as boundary conditions. This method is available in several commercial FEA codes, and is sometimes called "sub-modeling" [5]. Although "submodeling" can accurately model package stress effects, it can be tedious to implement and does not provide a "figure of merit" (FOM) for package stress.
A method similar to "sub-modeling" is proposed in this paper. This method approximates the results $(x, y$, and $z$ displacements) of the package model as functions of die location $(x$ and $y)$. These expressions have the form:

$$
U_{x}=C_{x m n} x^{m} y^{n} \quad U_{y}=C_{y m n} x^{m} y^{n} \quad \text { Eqn. }
$$

Eqn. 2

Where $\mathrm{Ux}, \mathrm{Uy}$, and $\mathrm{Uz}$ are displacements from the package model, and $x, y$ are the coordinates in the package model. The parameters (coefficients) $\mathrm{C}_{\mathrm{xmn}}, \mathrm{C}_{\mathrm{ymn}}$, and $\mathrm{C}_{\mathrm{zmn}}$ are found with curve fits of package model results. As an example $\mathrm{C}_{\mathrm{z} 20}$ is used to calculate the $\mathrm{z}$-axis displacement proportional to the term $x^{2} y^{0}$. In many cases package model displacements can be approximated with a small number (1-6) of coefficients.

Parametric representation of package stress effects provides several advantages. It provides a method of linking package and device models so that package effects can be estimated. The parameters used to represent substrate deformation provide a relatively simple, quantitative way of evaluating both packages (how much substrate deformation a given package produces) and devices (how sensitive to a given package stress a device is). Finally, these parameters can provide a means of communication between package and device designers, facilitating parallel development of packages and devices.

\section{MODELING PROCEDURE}

The first step in modeling is to divide the system into device and package FEA models; this division is based on relative stiffness. The relatively small, movable MEMS structures form the MEMS device model, and the remainder of components form the package model (Figure 1). Note that the silicon die ("substrate") that the MEMS device is fabricated on is in the package model. This is counter to general convention, and is necessary because the substrate is comparable in scale (and hence stiffness) to the other package components.

The package FEA model is then run with the appropriate loading. Possible loadings include thermal stresses from manufacture at elevated temperatures, thermal stresses from specified operating temperature, or external stresses (e.g. mechanical stresses from mounting the device on a circuit board). As an example, if Temperature Coefficient of Sensitivity (TCS) was modeled, the package model would be 
run at several different temperatures, to evaluate package stress vs. temperature.

Displacements from the package model are then fit with variations of Eqn. 1 and 2 (note: $x, y$, and $z$ directions are defined in Figure 1, with the $\mathrm{z}$ direction defined as normal to the substrate die.). This fit should be performed over the region of interest, i.e. the region on the substrate where the device is anchored. Possible package displacement modes include:

\section{Rigid Body Motion Modes:}

These terms can be ignored as they represent a movement of the entire package-device combination. As an example, if the entire die moves upward $1 \mu$, the device will move identically, and no stress or relative displacements will result. Rigid body motion translation can be expressed by equations of the form:

$$
U_{x}=C_{x 00}
$$

Rigid body rotation about the $\mathrm{x}$ and $\mathrm{y}$ axes can be expressed by equations of the form:

$$
U_{z}=C_{z 10} x
$$

Eqn. 4

In-plane Displacement, "Stretching Modes":

This mode assumes a uniform strain in the substrate. This can be expressed by the equation:

$$
\begin{array}{ll}
U_{x}=C_{x 10} x & \text { Eqn. } 5 \\
U_{y}=C_{y 01} y & \text { Eqn. } 6
\end{array}
$$

In this case displacement is directly proportional to location. This is a constant strain with the coefficients $C_{x 10}$ and $\mathrm{C}_{\mathrm{y} 01}$ analogous to the $\mathrm{x}$ and $\mathrm{y}$ strains respectively. If the two constants are equal the substrate is subjected to isotropic strain with no shear strain [6]. The "Stretching mode" can cause axial stresses in tethers that support moving structures, and this can cause "stress stiffening", affecting spring constants [7].

\section{"Bending Modes":}

The following equation describes second order, "out of plane" displacements in the substrate. These are called "bending modes":

$$
U_{z}=C_{z 20} x^{2}+C_{z 02} y^{2}
$$

Eqn. 7

This second order (parabolic) form approximates constant (circular) bending if the amount of displacement is small compared to the length of the surface (top of substrate). If the two constants are equal the substrate surface will have a spherical shape, with the radius of curvature approximated by $0.5 / C_{, 2(1)}$. MEMS devices that rely on nanometer scale vertical displacements can be sensitive to this mode.

\section{Other Modes:}

Other modes may be required to accurately characterize substrate displacements in the package model. The following equation describes one form of "twisting":

$U_{z}=C_{z l 1} x y$

Eqn. 8

Figures 2, 3, and 4 show actual examples of how package FEA model displacement data can be fit with equations 5, 6 , and 7. Figures 2 and 3 show that a parabolic curvature provides a good approximation of substrate bending. Figure 4 shows a linear dependence between $x$-displacement $x$ coordinate, indicating nearly constant value strain in this direction.

These parameters are then passed to the device model, where they are used to calculate boundary conditions. Several commercial FEA codes have features that allow automated input of boundary conditions, e.g. node displacements can be calculated as a function of location, as per eqn. $3-6$. The device model is then run, and the effects of different package induced displacements can then be evaluated.

The MEMS device often has a significant Coefficient of Thermal Expansion (CTE). Polysilicon, for example has a CIE about that of single crystal silicon [8]. Thus it is important to also model thermal strains in the device model. Omitting thermal strains in the device model is equivalent to using a MEMS device material with a CTE of zero, and this will generate unrealistic stresses in the device model. In the case of polysilicon structures, the ideal package should have thermal strains identical to those of silicon.

The package and MEMS device models can also be run in isolation. Thus several package options can be run to see which produces the least substrate deformation. Conversely several MEMS device models can be compared with identical package stress parameters to see which device is least sensitive to package stress.

\section{EXPERIMENTAL RESULTS}

Several packaging configurations were tested for the production, z-axis, $50 \mathrm{~g}$ accelerometer. The packaged sensor consisted of two dice (one capacitive device die and one control die) packaged in a PDIP package (see Figure 5). This package used an Alloy 42 leadframe, gold wire bonds, and was overmolded with a thermoset polymer. An elastomeric die bond and die coat were used to help isolate the device die from package stresses. The device die had a surface micromachined, polysilicon, z-axis accelerometer, and used a proof mass suspended by four tethers. The CMOS control die was used to produce a 0.5 to $4.5 \mathrm{~V}$ output [9], [10].

One package configuration caused accelerometer output to drift when stored at room temperature. Typically offset (zero acceleration reading) would shift upward, and sensitivity 
would drift downward. Similar output shifts occurred when this package configuration was externally clamped.

Both package and device FEA models were generated using ANSYS 5.3. The package model indicated that separation of one of the polymeric interfaces could cause significant changes in substrate deformation, especially in the "stretching mode" [11] (see eqn. 5 and 6). These changes in substrate strain were then expressed in parametric form and passed to the device model of the accelerometer, and used as boundary conditions. The device model was used to evaluate $\mathbf{z}$ axis displacements, and these displacements were used to calculate accelerometer output. Figure 6 shows the two parameter shifts (offset and sensitivity) for two experimental cases, and for the model. The model was in reasonable agreement with experimental data [12]. Both experimental and model data indicated that for this package configuration a $1 \%$ decrease in sensitivity is accompanied by approximately an $18 \mathrm{mV}$ increase in offset voltage. This modeling established that this interface separation was a possible cause of output shift in this package configuration.

\section{CONCLUSIONS}

Package stress effects can be efficiently modeled by using separate package and device FEA models. The package model can be run separately, and the resulting package stress (substrate deformation) data can be transferred to the device model in parametric form. These parameters provide a relatively simple way of quantifying package and device performance, and are suggested as a way of evaluating candidate package and device designs. This technique was used to model a package configuration for a capacitive, z-axis accelerometer, and model and experimental results were in approximate agreement.

\section{ACKNOWLEDGMENTS}

Many people provided help with this paper. These included Milena Vujosevic, Jonathan Hammond, Dan Koury, Gary O'Brien, Gary Li, Dave Monk, and Mike Saltmarsh.

\section{REFERENCES}

[1] G. Beardmore, "Packaging for Microengineered Devices. Lessons from the Real World," IEEE Colloquium on Assembly' and Connection in Microsystems, pp. 2/1-8 (1997)

[2] T. Dickerson, M. Ward, "Low Deformation and Stress Packaging of Micro-Machined Devices," IEEE Colloquium on Assembly and Connection in Microsystems, (Ref. No.1997/004) pp. 7/1-3 (1997)

[3] A. D. Romig, P. V. Dressendorfer, D. W. Palmer, "High Performance Microsystem Packaging: A Perspective," Microelectronic Reliability, Vol. 37, No. 1011, pp. 17711781 (1997).

[4] F. Pourahmadi, K. Peterson, "Package Design of Silicon Micromachined Sensors Using Finite Element Modeling,"
The 7th Annual Conference on Solid-State Sensors and Actuators, (1993)

[5] ANSYS Procedure Manual, Revision 5.3., Swanson Analysis Systems, Inc. (1996)

[6] A. Higdon et al., "Mechanics of Materials, third edition," John Wiley and Sons (1976)

[7] W. C. Young, "Beam under simultaneous Axial and Transverse Loading," Roarks Formulas for Stress and Strain, 6th Edition, pp. 156-181, McGraw Hill (1989)

[8] D. W. Burns, "Micromechanics of Integrated Sensors and the Planar Processed Pressure Transducer," Phd. thesis, University of Wisconsin (1988)

[9] F. Shemansky, et al., "A Two-Chip Accelerometer System for Automotive Applications," Microsystem Technologies, Vol. 1, No. 3, p. 121 (1995).

[10] D. Dougherty, Q. Li, M. Shah, "Sensor Package Design Improvements Using Computer Simulation and Experimental Testing," Sensors in Electronic Packaging (ASME 1995 Congress), MED-Vol. 3, pp. 11-17 (1995).

[11] M. Vujosevic, M. Shah, "Mechanical Causes of the Shift in the Sensitivity and Offset of the Medium g Accelerometer in PDIP - Impact of Dome Coat Coverage, " Proceedings of the 1998 Winter Motorola AMT Symposium, January 21\&22, Phoenix, AZ, pp. 1.36 - 1.43 (1998)

[12] M. Saltmarsh, Motorola internal correspondence, Oct. 2 (1997)

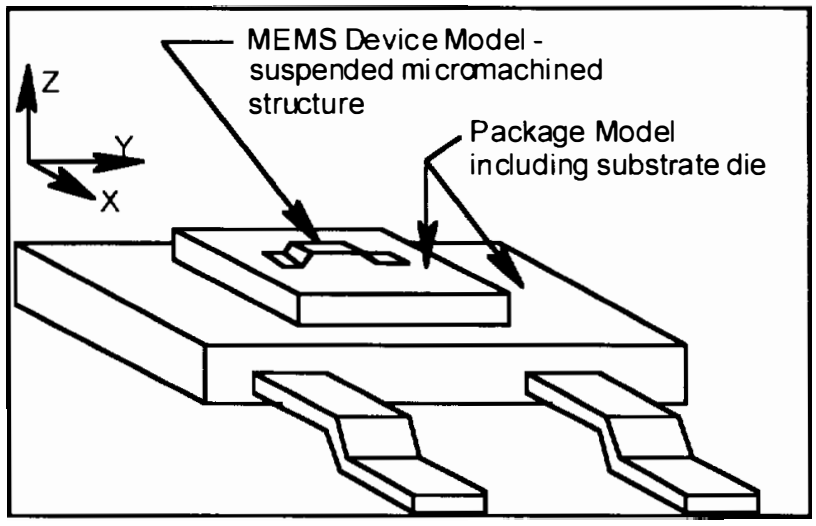

Figure 1: Schematic how a MEMS part is divided into two separate models. The package model includes conventional packaging plus the substrate die that MEMS device is fabricated on. The MEMS device model is comprised of the thin (micron range) micromachined structures. 


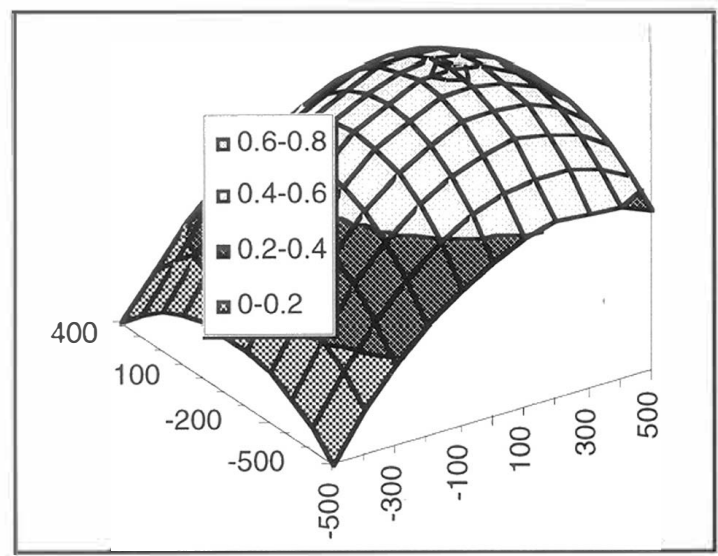

Figure 2: Z-displacement vs. die location (all units in microns) from package a FEA model, with Z-Displacement shown in the legend. This graph shows the curvature on the top surface of the device die.

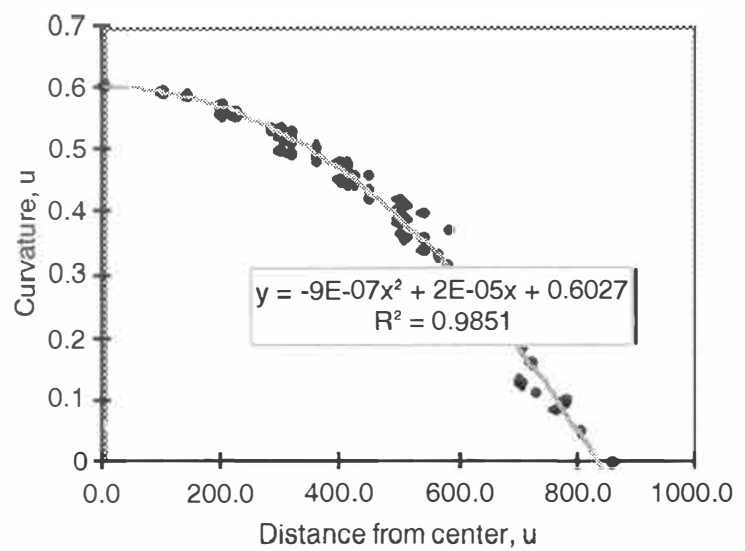

Figure 3: Z-displacements from Figure 2, plotted vs. radial distance from center of curvature. This data shows a good fit to a second order curve.

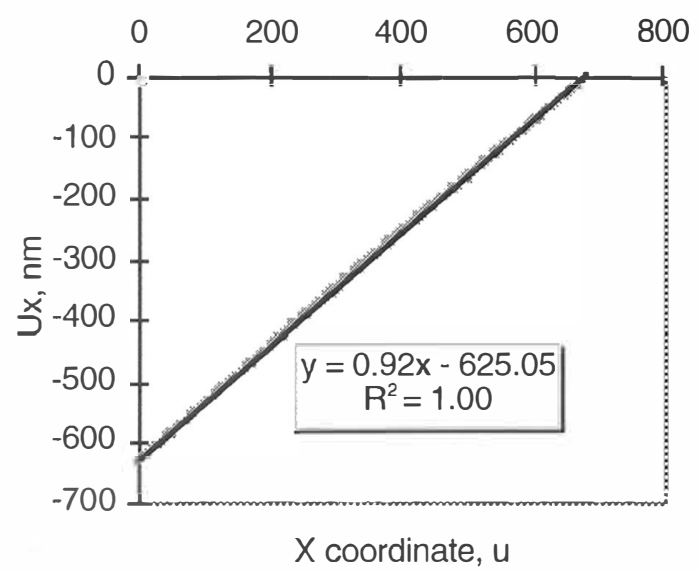

Figure 4: Package model, $x$-displacement as a function of die location, $x$-coordinate. The linear displacement function indicates constant strain, with the slope of the line equal to the strain.

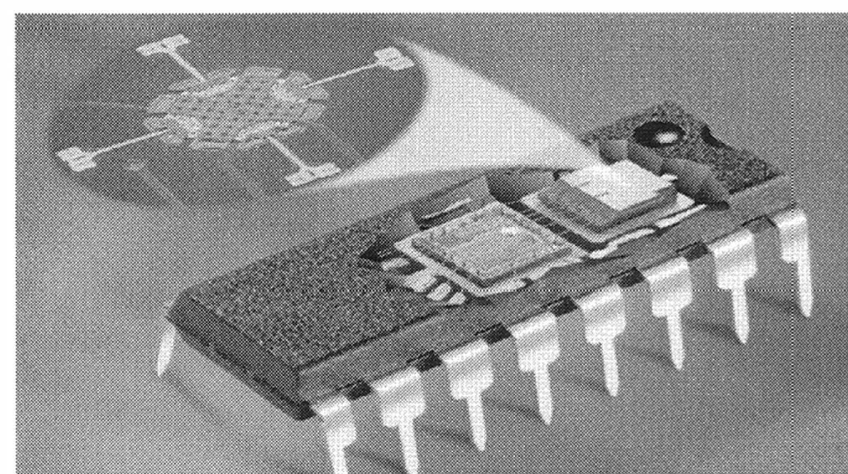

Figure 5: The z-axis accelerometer used for testing. Two dice (from left to right, control die and device die) are packaged in 16 pin, epoxy over-molded, PDIP package. A magnified view of the device die is shown. Note the proof mass suspended by four tethers.

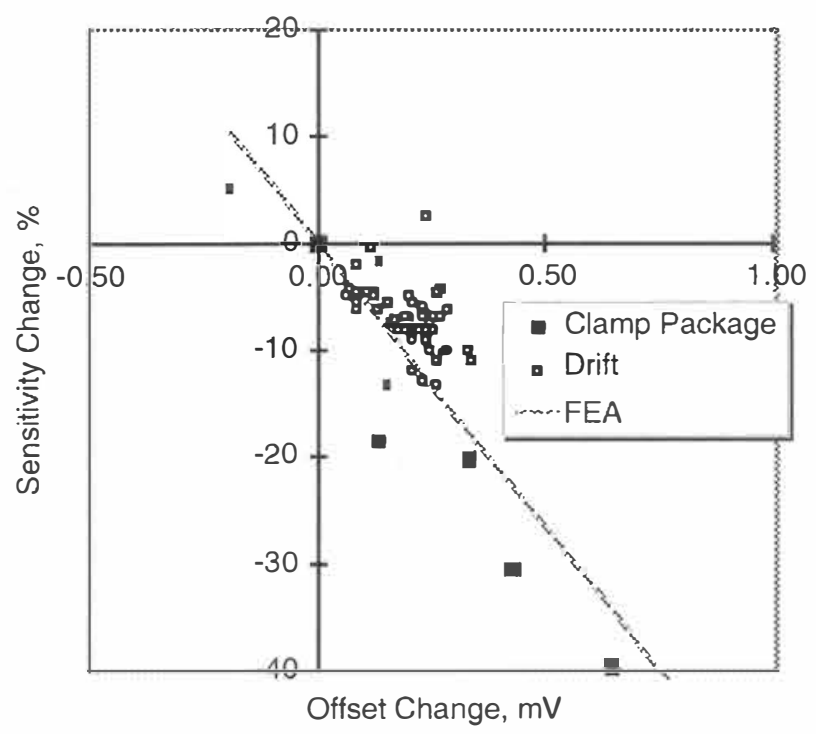

Figure 6: Comparison of FEA and Experimental Results, $40 \mathrm{~g} \mathrm{z}$-axis accelerometer in a plastic PDIP package. This graph shows the relationship between two parametric shifts: Offset shift and sensitivity shift. "Drift" data shows changes in accelerometer output during room temperature storage. "Clamp" data shows changes in output that occur when the packaged device is externally clamped. Both experimental and modeling data show a similar relationship between these two parametric shifts. 